\title{
Re-exposure to the hypobaric hypoxic brain injury of high altitude: plasma S100B levels and the possible effect of acclimatisation on blood-brain barrier dysfunction
}

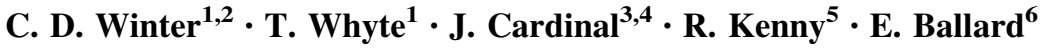

Received: 27 April 2015/Accepted: 15 February 2016/Published online: 29 February 2016

(C) The Author(s) 2016. This article is published with open access at Springerlink.com

\begin{abstract}
Hypobaric hypoxic brain injury results in elevated peripheral S100B levels which may relate to bloodbrain barrier (BBB) dysfunction. A period of acclimatisation or dexamethasone prevents altitude-related illnesses and this may involve attenuation of BBB compromise. We hypothesised that both treatments would diminish the S100B response (a measure of BBB dysfunction) on reascent to the hypobaric hypoxia of high altitude, in comparison to an identical ascent completed $48 \mathrm{~h}$ earlier by the same group. Twelve healthy volunteers, six of which were prescribed dexamethasone, ascended Mt Fuji (summit $3700 \mathrm{~m}$ ) and serial plasma S100B levels measured. The S100B values reduced from a baseline $0.183 \mu \mathrm{g} / 1$ (95 \% CI $0.083-0.283)$ to $0.145 \mu \mathrm{g} / \mathrm{l}(95 \% \mathrm{CI} 0.088-0.202)$ at high altitude for the dexamethasone group $(n=6)$ and from $0.147 \mu \mathrm{g} / 1(95 \% \mathrm{CI} 0.022-0.272)$ to $0.133 \mu \mathrm{g} / 1$ (95\% CI $0.085-0.182)$ for the non-treated group $(n=6)$ [not statistically significant $(p=0.43$ and $p=0.82)$ for the treated and non-treated groups respectively]. [These results contrasted with the statistically significant increase during
\end{abstract}

C. D. Winter

craigdw1@gmail.com

1 Kenneth Jamieson Department of Neurosurgery, Royal Brisbane and Women's Hospital, Herston, QLD, Australia

2 University of Queensland Centre for Clinical Research, Brisbane, QLD, Australia

3 Department of Chemical Pathology, Royal Brisbane and Women's Hospital, Herston, QLD, Australia

4 School of Human Movement Studies, University of Queensland, St Lucia, QLD, Australia

5 CAE Solutions, Iidabashi, Chiyoda-Kyu, Tokyo, Japan

6 Queensland Institute of Medical Research, Herston, QLD, Australia the first ascent, S100B increasing from $0.108 \mu \mathrm{g} / 1$ (95\% CI $0.092-0.125)$ to $0.216 \mu \mathrm{g} / 1(95 \%$ CI $0.165-0.267)$ at high altitude]. In conclusion, an increase in plasma S100B was not observed in the second ascent and this may relate to the effect of acclimatisation (or hypoxic pre-conditioning) on the BBB. An exercise stimulated elevation of plasma S100B levels was also not observed during the second ascent. The small sample size and wide confidence intervals, however, precludes any statistically significant conclusions and a larger study would be required to confirm these findings.

Keywords S100B $\cdot$ Hypoxia $\cdot$ High altitude $\cdot$ Bloodbrain barrier $\cdot$ Dexamethasone $\cdot$ Acclimatisation

\section{Introduction}

$\mathrm{S} 100 \mathrm{~B}$ is a $21 \mathrm{kDa}$ dimeric calcium-binding protein predominantly found in brain astrocytes [1] which regulates a variety of cellular functions [2]. There is an extensive body of literature regarding clinical conditions that have been associated with elevated S100B levels. A recent review [3] describes S100B associations with psychiatric disorders (schizophrenia, depression), sport (running, boxing, soccer), tumours (melanoma), multiple sclerosis, Alzheimer's disease, cardiac disease, stroke and patients with traumatic brain injury or multi-trauma in the absence of brain injury. Peripheral S100B levels have also been noted to increase in patients with other hypoxic conditions such as obstructive sleep apnoea [4] and its postulated neuroprotective properties may also reflect increased expression secondary to post-injury reparative processes [5, 6]. Despite this many authors believe that peripheral S100B levels correlates with blood-brain barrier (BBB) dysfunction [7-9]. That CSF- 
serum albumin quotient (QA) is considered to be the gold standard for quantifying the extent of BBB dysfunction [10] and good correlation with serum S100B levels have been reported [9] provides further supportive data.

Although high-altitude related illnesses, such as acute mountain sickness (AMS), are thought to result from a multitude of processes, it is considered that blood-brain barrier (BBB) disruption is a significant contributor [11, 12]. Elevated peripheral S100B levels have been confirmed in volunteers at high altitude and this may reflect hypoxic opening of the BBB [13-15]. Furthermore, the vascular permeability factor vascular endothelial growth factor (VEGF) affects BBB function by altering tight junctions between endothelial cells and its increased expression in hypoxic conditions has been confirmed in animal studies [16] and mountaineers at high altitude [17]. The hypobaric hypoxia associated with altitude gain may induce VEGF release, a compromised BBB, cerebral vasogenic oedema and elevation of peripheral S100B. MRI findings of mild extracellular vasogenic oedema at simulated high altitude has also been described, suggestive of BBB compromise [18].

During the process of acclimatisation individuals exhibit specific cerebral physiological adaptive responses to reduced oxygen levels, such as increased cerebral blood flow (CBF) [19] and intracranial pressure [11]. Improved exercise tolerance, cognitive performance and symptoms of AMS following 16 days at high altitude have been reported [20]. Of more importance the latter group noted that after a period at low altitude the reduced incidence of AMS persisted upon re-ascent. Wu et al. [21] also reports improved AMS scores during re-exposure to $4500 \mathrm{~m}$ following long periods (up to 5 months) of low altitude, which correlated with improved oxygen saturations and lower resting HR. To our knowledge BBB dysfunction during re-exposure to high altitude has not been previously investigated. We hypothesised that the improvement in altitude related illnesses following acclimatisation may result from a beneficial effect on the BBB and this may correlate with diminished S100B levels. Our first paper [14] served to document two important stand-alone conclusions; that a statistically significant elevation in plasma S100B occurs with altitude but the S100B levels did not correlate with AMS. This companion paper from the same individuals at the same time points during the same ascent $48 \mathrm{~h}$ earlier allows us to compare two S100B data sets and comment on any possible effects of acclimatisation on S100B levels. When the participants ascended Mt Fuji $48 \mathrm{~h}$ earlier (i.e. the first ascent) they commenced at the same time of day, carried the same amount of weight (batteries, dry ice, medical equipment, etc.), took the same time interval to complete the distances between bleed-points, stayed overnight at the summit (exactly as per the second ascent) and descended at the same rate. We therefore felt that the two S100B data sets were comparable.

Dexamethasone is used for the prophylaxis and treatment of AMS and high altitude cerebral edema (HACE) [12] and this may involve attenuation of the BBB. The exact mechanism is unclear, though may involve changes in calcium-activated potassium channels [22] or reducing tight junction [23] or VEGF expression [24, 25]. We further hypothesised that volunteers prescribed dexamethasone would exhibit an additional reduction in S100B, in comparison to both the first ascent and with those who did not take dexamethasone in the second ascent. Studying the release of S100B in subjects at high altitude comprises a method of evaluating the effect of reversible hypoxic injury on the human BBB. Brain imaging (MRI) has been used in studies of simulated high altitude but clearly is not available to investigate the brain response at actual high altitude. Insights into the molecular responses by the brain to the hypobaric hypoxia of high altitude and re-exposure may potentially provide support for the development of novel therapeutic strategies for high attitude related illnesses and other hypoxic brain injuries.

As an addendum, a potential confounding factor for any such field study is the relationship between peripheral S100B levels and strenuous exercise. A recent systematic review [26] lists several studies confirming higher S100B levels post-exercise compared to pre-exercise values but also reports many studies which failed to confirm such a relationship. Ascending to $3700 \mathrm{~m}$ involves significant exertion and by comparing the two ascents we may also be able to comment on the possible phenomenon of exercise induced S100B elevation.

\section{Method}

Following consent 12 healthy volunteers (gender ratio 1:1, age range 22-56) were recruited. The Lake Louise Questionnaire [27] and oxygen saturations (TuffSat, Datex Ohmeda) were completed at each bleed point. AMS was diagnosed when the participant experienced a headache and one other symptom to yield a score of 4 or more. On day 2 the group was driven to $1400 \mathrm{~m}$, trekked to $2590 \mathrm{~m}$ (second blood test), and continued to the overnight accommodation at $3200 \mathrm{~m}$. On day 3 we reached the summit $(3700 \mathrm{~m})$, remained for $2-3 \mathrm{~h}$ (blood test) and descended the mountain (blood test at $2590 \mathrm{~m}$ ). Regular fluid intake was ensured in order to prevent dehydration. In summary, samples for S100B were taken at baseline $(32 \mathrm{~m}$ above sea level), at 2590, at 3700 and then again at $2590 \mathrm{~m}$ during descent. The 12 subjects were randomly divided into a dexamethasone $(n=6)$ and a non-dexamethasone group $(n=6)$. The standard dose for the prevention or 
treatment of AMS was used ( $4 \mathrm{mg}$ twice daily for $2 \frac{1}{2}$ days commencing at the beginning of the ascent).

At each bleed point $12 \mathrm{ml}$ of venous blood (Li-Heparin tubes) was centrifuged for $3 \mathrm{~min}$ at $7200 \mathrm{rpm}$ (4400g) using a portable centrifuge (StatSpin X3) (powered by $12 \mathrm{~V}$ batteries connected to an AC/DC inverter). The plasma was placed into dry ice $\left(-80^{\circ}\right)$, transported to Australia, stored at $-80^{\circ}$ and analyzed in duplicate with an automated chemiluminescence immunoassay (DiaSorin Liaison, Stillwater, MN, USA) with the Sangtec ${ }^{\circledR}$ S100 analysis kit. For some of the participants the level at $2590 \mathrm{~m}$ was actually higher than the $3700 \mathrm{~m}$ level during ascent. The $2590 \mathrm{~m}$ value followed a fairly rapid ascent from 1400 to $2590 \mathrm{~m}(1190 \mathrm{~m}$ in $6-8 \mathrm{~h})$ and it is well recognised that altitude sickness (and therefore potentially hypoxia induced BBB compromise and S100B level) relates to the rate of ascent as well as the final altitude gained. In addition, the $3700 \mathrm{~m} \mathrm{S100B}$ level followed a period of rest at $3200 \mathrm{~m}$ after which we ascended to the summit and rested again for $2-3 \mathrm{~h}$ prior to the sample being taken. This summit S100B level may therefore have reflected a period of acclimatisation. Similarly, the $2590 \mathrm{~m}$ level during descent was taken within a few hours of the maximal period of hypobaric hypoxia on the summit. It was therefore deemed appropriate to use a mean of the three altitudes (2590 and 3700 on ascent, $2590 \mathrm{~m}$ on descent) for all subjects. The plasma S100B level was summarised by calculating a mean with $95 \%$ confidence intervals at baseline and at high altitude.

Student's $t$ tests for independent or matched samples were used to compare groups or time points, respectively. The study has been reviewed and approved by the Royal Brisbane and Women's Hospital Human Ethics Research Committee and carried out according to the National Statement on Ethical Conduct in Human Research (2007) produced by the National Health and Medical Research Council of Australia.

\section{Results}

The results from this second ascent are presented and contrast with the parallel S100B data from the first ascent for the same 12 people [14]. The results for this current paper (the second ascent) note that 6 of the 12 participants were treated with dexamethasone (the 'treated group'). The plasma S100B values reduced from $0.183 \mu \mathrm{g} / \mathrm{l}(95 \% \mathrm{CI}$ $0.083-0.283)$ at baseline to $0.145 \mu \mathrm{g} / 1 \quad(95 \%$ CI 0.088-0.202) at altitude for the dexamethasone-treated group and from $0.147 \mu \mathrm{g} / \mathrm{l}(95 \%$ CI $0.022-0.272)$ at baseline to $0.133 \mu \mathrm{g} / \mathrm{l}(95 \%$ CI $0.085-0.182)$ at altitude for the non-treated group (Table 1 ). The reductions in $\mathrm{S} 100 \mathrm{~B}$ between baseline and altitude for this second ascent were not statistically significant for the treated $(p=0.43$ ) or non-treated ( $p=0.82$ ) groups. There were no significant differences in plasma S100B levels at either baseline ( $p=0.57)$ or at altitude $(p=0.70)$ between the treated or non-treated groups. Furthermore there was no statistically significant difference in the baseline plasma S100B level between the two ascents [all patients $p=0.077$, treated $p=0.22$, non-treated $p=0.27]$. To summarise the first ascent $\mathrm{S} 100 \mathrm{~B}$ results, there was a significant $(p<0.001)$ increase in plasma S100B level from $0.108 \mu \mathrm{g} / \mathrm{l}(95 \% \mathrm{CI}$ $0.092-0.125)$ at baseline to $0.216 \mu \mathrm{g} / \mathrm{l} \quad(95 \% \mathrm{CI}$ $0.165-0.267$ ) at high altitude.

During this ascent there was a statistically significant decrease in oxygen saturations from baseline to altitude for both the treated group [baseline $97.3 \% \quad(95 \%$ CI 95.9-98.8), at altitude $92.2 \%$ (95\% CI 89.9-94.4), $p=0.001$ ] and for the non-treated group [baseline $97.8 \%$ $(95 \%$ CI $97.4-98.3)$ at altitude $92.7 \% \quad(95 \%$ CI 90.4-94.9), $p=0.004]$. There were no significant differences at either baseline $(p=0.41)$ or at altitude $(p=0.70)$ in oxygen saturations between the treated and non-treated groups. There was no statistically significant difference in the baseline oxygen saturation levels between the two ascents (all participants $p=0.62$, treated $p=0.46$, untreated $p=0.36$ ).

For completeness we compared the incidence of and S100B levels for AMS and non-AMS subjects. In the first ascent seven subjects experienced acute mountain sickness. During the second ascent the incidence of AMS reduced, affecting only three subjects (one from the dexamethasone group and two from the non-treated group), indicating that the process of acclimatisation had occurred. No correlation between plasma S100B levels and acute mountain sickness was evident during the first ascent and the same lack of correlation between S100B and AMS was found for the second ascent (Table 2). Given that there is no significant difference in plasma S100B levels between treatments and insufficient numbers of participants with AMS, comparisons were made between AMS and non-AMS sufferers only. The plasma S100B levels for the three AMS sufferers changed from $0.100 \mu \mathrm{g} / \mathrm{l}(95 \% \mathrm{CI}-0.029$ to 0.229$)$ at baseline to $0.127 \mu \mathrm{g} / \mathrm{l}(95 \% \mathrm{CI}-0.031$ to 0.284$)$ at high altitude which was marginally significant $(p=0.057)$ compared to the non-AMS sufferers who showed a nonsignificant $(p=0.35)$ change from $0.187 \mu \mathrm{g} / 1$ (95\% CI $0.102-0.272)$ at baseline to $0.143 \mu \mathrm{g} / \mathrm{l} \quad(95 \%$ CI 0.108-0.179) at altitude. There was no significant difference in plasma S100B levels between AMS and non-AMS groups at baseline $(p=0.23)$ or at altitude $(p=0.63)$. There was no significant difference in oxygen saturations between AMS and non-AMS participants at baseline $(p=0.88)$ or at altitude $(p=0.49)$. There was no significant difference between oxygen saturation levels at 
Table 1 Plasma S100B ( $\mu \mathrm{g} / \mathrm{l})$ and oxygen saturations (\%) during re-ascent of Mt Fuji (3700 m)

\begin{tabular}{|c|c|c|c|c|}
\hline Parameter & Group & $\begin{array}{l}\text { Baseline [mean } \\
(95 \% \mathrm{CI})]\end{array}$ & $\begin{array}{l}\text { Average at altitude } \\
\text { [mean }(95 \% \mathrm{CI})]\end{array}$ & $\begin{array}{l}p \text { value between } \\
\text { altitudes }\end{array}$ \\
\hline \multirow[t]{2}{*}{ Plasma S100B $(\mu \mathrm{g} / \mathrm{l})$} & Treated $(n=6)$ & $0.183(0.083-0.283)$ & $0.145(0.088-0.202)$ & 0.43 \\
\hline & Nontreated $(n=6)$ & $0.147(0.022-0.272)$ & $0.133(0.085-0.182)$ & 0.82 \\
\hline$p$ value between treatments & & 0.57 & 0.70 & \\
\hline \multirow[t]{2}{*}{ Oxygen saturation (\%) } & Treated $(n=6)$ & $97.3(95.9-98.8)$ & $92.2(89.9-94.4)$ & 0.001 \\
\hline & Nontreated $(n=6)$ & $97.8(97.4-98.3)$ & $92.7(90.4-94.9)$ & 0.004 \\
\hline$p$ value between treatments & & 0.41 & 0.70 & \\
\hline
\end{tabular}

Treated dexamethasone treated group, nontreated non-dexamethasone group

Table 2 Plasma S100B and oxygen saturations (\%) during re-ascent of Mt Fuji (3700 m); comparison of AMS and non-AMS subjects

\begin{tabular}{lllll}
\hline Parameter & Group & $\begin{array}{l}\text { Baseline [mean } \\
(95 \% \mathrm{CI})]\end{array}$ & $\begin{array}{l}\text { Average at altitude } \\
\text { [mean }(95 \% \text { CI })]\end{array}$ & $\begin{array}{l}p \text { value between } \\
\text { altitudes }\end{array}$ \\
\hline Plasma S100B $(\mu \mathrm{g} / \mathrm{l})$ & AMS $(n=3)$ & $0.100(0.029-0.229)$ & $0.127(-0.031$ to 0.284$)$ & 0.057 \\
& Non-AMS $(n=9)$ & $0.187(0.102-0.272)$ & $0.143(0.108-0.179)$ & 0.35 \\
$p$ value between treatments & & 0.23 & 0.63 & $91.7(86.5-96.8)$ \\
Oxygen saturation $(\%)$ & AMS $(n=3)$ & $97.7(96.2-99.1)$ & $92.7(91.0-94.3)$ & $<0.001$ \\
$p$ value between treatments & Non-AMS $(n=9)$ & $97.6(96.7-98.4)$ & 0.49 & \\
\hline
\end{tabular}

Treated dexamethasone treated group, nontreated non-dexamethasone group, $A M S$ acute mountain sickness

baseline between ascents for the non-AMS sufferers $(p=0.45)$ and AMS sufferers $(p=81)$.

\section{Discussion}

An elevation of peripheral S100B from baseline following the hypobaric hypoxia of high altitude has been previously documented and is thought to represent hypobaric hypoxic induced opening of the blood-brain barrier [13, 15]. The statistically significant increase in plasma S100B during ascent to the same altitude using the same group of volunteers [14] $48 \mathrm{~h}$ earlier allows us to comment on the potential effects of acclimatisation on the BBB when we compare those S100B levels with this second ascent. Although an elevation in S100B was not observed in this (second) ascent during re-exposure to the hypobaric hypoxia of high altitude for either the non-treated (acclimatised) or the dexamethasone treated (acclimatised and dexamethasone) groups, the hypothesis that $\mathrm{S100B}$ would reduce following acclimatisation could not be statistically confirmed in our study given the wide confidence intervals and small sample size. A larger study would be required to confirm these findings.

It would be easier to explain a lack of hypoxia-induced $\mathrm{BBB}$ compromise in our subjects during re-exposure to hypoxia if higher oxygen saturations were present compared to the first ascent. The reported improved oxygen saturations during re-exposure to hypobaric hypoxia [21, $28,29]$ did not occur for our participants. The oxygen saturations at high altitude for the dexamethasone and the non-dexamethasone group were 92.2 and $92.7 \%$ respectively, compared to $91.6 \%$ for the first ascent, i.e. not significantly different (all patients $p=0.075$, treated $p=0.36$, untreated $p=0.14$ ). Hypothesising on the mechanism(s) that may be involved in the improved BBB dysfunction following re-exposure to high altitude, extraneous to improved oxygen saturation, is speculative but may involve cerebral autoregulation, increased chemosensitivity or hypoxic preconditioning. Cerebral autoregulation (CA), the process by which CBF is maintained despite fluctuations in cerebral perfusion pressure (CPP), is impaired at high altitude and Subudhi et al. [20] recently confirmed persistence of dysfunctional CA on re-ascent. A failure of autoregulation may result in passive cerebral vasodilatation, a compromised BBB and increased vasogenic oedema. The degree of altered autoregulation may have been less severe $48 \mathrm{~h}$ later in our participants resulting in an improved $\mathrm{BBB}$ relative to the first ascent. A concept of enhanced hypoxic chemosensitivity has been suggested which posits that intermittent periods of hypoxia results in an augmented ventilatory response on re-exposure $[30,31]$. A modified ventilation response to hypoxia or $\mathrm{CO}_{2}$ levels may theoretically adjust the balance of 
hypocapneic vasoconstriction versus hypoxic vasodilatation, local $\mathrm{pH}$ and bicarbonate concentrations. These dynamic processes all of which could potentially alter arteriolar and capillary vessel calibre and hydrostatic pressure changes with subsequent effects on the BBB during hypoxic re-exposure. The activation of specific genes is an important addition to adaptation to hypoxic conditions [31] and it may be that downstream gene products or transcription factors such as hypoxia-induciblefactor-1 (HIF-1) were still at play $48 \mathrm{~h}$ after being switched on and that an undiscovered mechanism attenuated the hypoxic induced BBB dysfunction. S100B has known neuro-protective properties, reducing glutamate excitotoxic damage [32] and enhancing neurogenesis [33] and the increased expression of S100B as part of a neuro-protective response to hypoxia may have been modified by acclimatisation.

Hypoxic pre-conditioning refers to the phenomenon of exposure to a moderate hypoxia/ischaemic (HI) insult providing increased resistance to a subsequent episode of $\mathrm{HI}[34,35]$. The first ascent may have acted as hypoxic preconditioning and this may have affected the BBB response to the second hypobaric hypoxic insult. Wick et al. [36] report an in vitro model enabling investigation into the possible molecular mechanisms involved in hypoxic preconditioning. Cerebellar granule neurons (CGN) preconditioned by sub-lethal exposure to reduced oxygen tension became resistant to successive injuries. The authors report elevated levels of VEGF and that the activation of VEGF/ VEGF-receptor-2 (VEGFR-2), coupled with Akt/protein kinase $\mathrm{B}$ (PKB) phosphorylation and the subsequent enhancement of anti-apoptotic proteins may be integral to the neuroprotection afforded by hypoxic preconditioning. The enhanced expression of VEGF may also directly affect BBB permeability by causing loss of tight junction (TJ) proteins (such as occludin and claudin-5) and subsequent endothelial barrier disruption [37]. Following hypoxic injury to the brain the onset of a neuro-inflammatory cascade develops leading to increased release of cytokines, chemokines, reactive oxygen species and matrix metalloproteinases, all of which may cause disruption of the BBB. Significant protection secondary to hypoxic preconditioning in an in vivo model of neonatal HI injury has been described [38]. The authors report that the underlying mechanism may involve suppression of pro-inflammatory gene expression and a reduction in glial cell activation and the subsequent inflammation following re-exposure to hypoxia. Furthermore, Tang et al. [39] used a mouse model to examine the specific genomic response to ischemia following hypoxic preconditioning and compared this to gene expression following ischemia alone. They report the up-regulation of 27 genes mediating diverse function as sodium homeostasis, aquaporin-4 water channel expression, stress response and pro-inflammatory genes, all of which could conceivably affect blood-brain barrier function in the preconditioned ischemic animals. Many of these processes may account for the lack of a pronounced S100B response (potentially reflecting improved BBB function) during the second hypobaric hypoxic stimulus of the second ascent.

Our results also allow comment on the potential effects of dexamethasone on the BBB. A recent meta-analysis has confirmed the benefit of dexamethasone in the treatment and prevention of high altitude illnesses [40]. We hypothesised that dexamethasone would attenuate the hypobaric hypoxia induced BBB 'leakiness' and this would manifest as a lower S100B level in the dexamethasone treated group. However, there was no difference in high altitude plasma S100B level between the dexamethasone treated $(n=6)$ and non-treated groups $(n=6)$, we therefore could not confirm an additive effect of the steroid on attenuating the BBB compromise. An alternative possibility is that the process of acclimatisation overshadowed any potential effect of dexamethasone on S100B levels.

For any field study such as this we have to consider the effect of strenuous exertion on peripheral biomarker levels. Koh et al. [26] provides a review with some studies correlating elevated S100B levels with exercise while others did not. During our ascent the strenuous physical exertion was not associated with an increased S100B discounting any association. Comparing the S100B levels from the first ascent to this study we can conclude that if the elevated plasma S100B level in the first ascent was related to physical exertion, then a similar rise would be expected during the second ascent, but this was not observed. Our subjects were instructed to drink ad libitum reducing any potential dehydration effects. For completeness we analysed the S100B data for AMS and non-AMS subjects in the second ascent (Table 2). These results were in agreement with the first ascent, i.e. no correlation between AMS and S100B levels.

This study is open to the criticisms of any field study. Studies recruiting additional participants, measuring other biomarkers of BBB (e.g. matrix metalloproteinases) and the use of a hypoxic chamber with equivalent exercise are warranted. The effect of dexamethasone (without acclimatisation) may have been more instructive if we had repeated the ascent in 6 months, but we were limited by volunteer availability and funding restrictions. With hindsight it may have been prudent to combine the results from both ascents but the increase in S100B with altitude and the lack of correlation with AMS were important stand-alone conclusions. Additionally our S100B results relate to an ascent to $3700 \mathrm{~m}$, and a more severe hypoxic stimulus (higher altitude) may have facilitated a statistical difference in the S100B levels between the 
dexamethasone treated and non-treated groups. Despite the limitations, to our knowledge the investigation of plasma S100B and by extension BBB function during reexposure to high altitude, has not been previously explored. Analysing the molecular response of the BBB to hypobaric hypoxia and re-exposure to high altitude may elucidate secondary mechanisms that are common to many forms of brain injury such as stroke or subarachnoid haemorrhage.

\section{Conclusion}

Our data suggests that the increase in plasma S100B during the first ascent was not observed in the second ascent for either the dexamethasone treated or non-treated groups. An exercise stimulated elevation of plasma S100B levels was also not observed during the second ascent. The small sample size and wide confidence intervals precludes any statistically significant conclusions and a larger study would be required to confirm these findings.

Acknowledgments To the Nursing Staff, their families and friends who volunteered for the study. The study was funded entirely by the Neurosurgical Research Trust Fund of the Royal Brisbane and Women's Hospital.

\section{Compliance with ethical standards}

Conflict of interest The authors have no conflict of interest

Open Access This article is distributed under the terms of the Creative Commons Attribution 4.0 International License (http://crea tivecommons.org/licenses/by/4.0/), which permits unrestricted use, distribution, and reproduction in any medium, provided you give appropriate credit to the original author(s) and the source, provide a link to the Creative Commons license, and indicate if changes were made.

\section{References}

1. Beaudeux J, Dequen L, Foglietti M (1999) Pathophysiologic aspects of S100 beta protein: a new biological marker of brain pathology. Ann Biol Clin (Paris) 57:261-272

2. Heizmann CW, Fritz G, Schafer BW (2002) S100 proteins: structure, functions and pathology. Front Biosci 1(7):d1356d1368

3. Michetti F, Corvino V, Geloso MC, Lattanzi W, Bernardini C, Serpero L, Gazzolo D (2012) The S100B protein in biological fluids: more than a lifelong biomarker of brain distress. J Neurochem 120(5):644-659

4. Braga CW, Martinez D, Wofchuk S, Portela LV, Souza DO (2006) S100B and NSE serum levels in obstructive sleep apnoea syndrome. Sleep Med 7:431

5. Kleindienst A, Bullock RM (2006) A critical analysis of the role of the neurotrophic protein S100B in acute brain injury. J Neurotrauma 23(8):1185-1200

6. Donato R, Sorci G, Riuzzi F, Arcuri C, Bianchi R, Brozzi F, Tubaro C, Giambanco I (2009) S100B's double life: intracellular regulator and extracellular signal. Biochim Biophys Acta 1793(6): 1008-1022

7. Kapural M, Krizanac-Bengez L, Barnett G, Perl J, Apollo D, Rasmussen P, Mayberg MR, Janigro D (2002) Serum S100beta as a possible marker of blood-brain barrier disruption. Brain Res 940:102-104

8. Marchi N, Rasmussen P, Kapural M, Fazio V, Kight K, Mayberg MR, Kanner A, Ayumar B, Albensi B, Cavaglia M, Janigro D (2003) Peripheral markers of brain damage and blood-brain barrier dysfunction. Restor Neurol Neurosci 21(3-4):109-121

9. Blyth BJ, Farhavar A, Gee C, Hawthorn B, He H, Nayak A, Stocklein V, Bazarian JJ (2009) Validation of serum markers for blood-brain barrier disruption in traumatic brain injury. J Neurotrauma 26:1497-1507

10. Reiber H, Peter JB (2001) Cerebrospinal fluid analysis: diseaserelated data patterns and evaluation programs. J Neurol Sci 184:101-122

11. Wilson MH, Newman S, Imray CH (2009) The cerebral effects of ascent to high altitudes. Lancet Neurol 8:175-191

12. Imray C, Wright A, Subudhi A, Roach R (2010) Acute mountain sickness: pathophysiology, prevention, and treatment. Prog Cardiovasc Dis 52(6):467-484

13. Bjursten H, Ederoth P, Sigurdsson E, Gottfredsson M, Syk I, Einarsson O, Gudbjartsson T (2010) S100B profiles and cognitive function at high altitude. High Alt Med Biol 11:31-38

14. Winter CD, Whyte TR, Cardinal J, Rose SE, O'Rourke PK, Kenny RG (2014) Elevated plasma S100B levels in high altitude hypobaric hypoxia do not correlate with acute mountain sickness. Neurol Res 36(9):779-785

15. Gao YX, Li P, Jiang CH, Liu C, Chen Y, Chen L, Ruan HZ, Gao YQ (2014) Psychological and cognitive impairment of long-term migrators to high altitudes and the relationship to physiological and biochemical changes. Eur J Neurol. doi:10.111/ene.12507

16. Feng Y, Rhodes PG, Bhatt AJ (2010) Hypoxic preconditioning provides neuroprotection and increases vascular endothelial growth factor A, preserves the phosphorylation of Akt-Ser-473 and diminishes the increase in caspase- 3 activity in neonatal rat hypoxic-ischemic model. Brain Res 14(1325):1-9

17. Dorward DA, Thompson AA, Ballie JK, MacDougall M, Hirani N (2007) Change in plasma vascular endothelial growth factor during onset and recovery from acute mountain sickness. Respir Med 101:587-594

18. Kallenberg K, Bailey DM, Christ S, Moir A, Roukens R, Menold E, Steiner T, Bartsch P, Knauth M (2007) Magnetic resonance imaging evidence of cytotoxic cerebral oedema in acute mountain sickness. J Cereb Blood Flow Metab 27:1064-1071

19. Ainslie PN, Subudhi AW (2014) Cerebral blood flow at high altitude. High Alt Med Biol 15(2):133-140

20. Subudhi AW, Fan JL, Evero O, Bourdillon N, Kayser B, Julian CG, Lovering AT, Panerai RB, Roach RC (2014) AltitudeOmics: cerebral autoregulation during ascent, acclimatization, and reexposure to high altitude and its relation with acute mountain sickness. J Appl Physiol 116:724-729

21. Wu TY, Ding SQ, Liu JL, Yu MT, Jia JH, Duan JQ, Chai ZC, Dai RC, Zhang SL, Liang BZ, Zhao JZ, Qi DT, Sun YF, Kayser B (2009) Reduced incidence and severity of acute mountain sickness in Qinghai-Tibet railroad construction workers after repeated 7-month exposures despite 5-month low altitude periods. High Alt Biol Med 10:221-232

22. Gu YT, Qin LJ, Qin X, Xu F (2009) The molecular mechanism of dexamethasone-mediated effect on blood-brain barrier permeability in a rat brain tumor model. Neurosci Lett 452(2):114-118

23. Sadowski GB, Malaeb SN, Stonestreet BS (2010) Maternal glucocorticoid exposure alters tight junction protein expression in the brain of fetal sheep. Am J Physiol Heart Circ Physiol 298(1):H179-H188 
24. Fischer S, Renz D, Schaper W, Karliczek GF (2001) In vitro effects of dexamethasone on hypoxia-induced hyperpermeability and expression of vascular endothelial growth factor. Eur $\mathbf{J}$ Pharmacol 411:231-243

25. Kim H, Lee JM, Park JS, Jo SA, Kim YO, Kim CW, Jo I (2008) Dexamethasone co-ordinately regulates angiopoietin-1 and VEGF: a mechanism of glucocorticoid-induced stabilization of blood-brain barrier. Biochem Biophys Res Commun 372(1):243-248

26. Koh SXT, Lee JKW (2014) S100B as a marker of brain damage and blood-brain barrier disruption following exercise. Sports Med 44:369-385

27. Roach RC, Bartsch P, Oelz O, Hackett P (1993) The Lake Louise acute mountain sickness scoring system. In: Sutton JR, Houston CS, Coates G (eds) Hypoxia and molecular medicine. Queen City Press, Burlington, pp 272-274

28. MacNutt MJ, Laursen PB, Kedia S, Neupane M, Parajuli P, Pokharel J, Sheel AW (2012) Acclimatisation in trekkers with and without recent exposure to high altitude. Eur J Appl Physiol 112:3287-3294

29. Subudhi AW, Bourdillon N, Bucher J, Davis C, Elliott JE, Eutermoster M, Evero O, Fan JL, Jameson-Van Houten S, Julian CG, Kark J, Kark S, Kayser B, Kern JP, Kim SE, Lathan C, Laurie SS, Lovering AT, Paterson R, Polaner DM, Ryan BJ, Spira JL, Tsao JW, Wachsmuth NB, Roach RC (2014) AltitudeOmics: the integrative physiology of human acclimatization to hypobaric hypoxia and its retention upon reascent. PLoS ONE 9(3):e92191. doi:10.1371/journal.pone.0092191

30. Katayama K, Sato Y, Morotome Y, Shima N, Ishida K, Mori S, Miyamura M (2001) Intermittent hypoxia increases ventilation and $\mathrm{SaO}_{2}$ during hypoxic exercise and hypoxic chemosenstivity. J Appl Physiol 90:1431-1440

31. Prabhakar NR, Kline DD (2002) Ventilatory changes during intermittent hypoxia: importance of pattern and duration. High Alt Med Biol 1:195-204
32. Tramontina F, Tramontina AC, Souza DF, Leite MC, Gottfried C, Souza DO, Wofchuk ST, Goncalves CA (2006) Glutamate uptake is stimulated by extracellular S100B in hippocampal astrocytes. Cell Mol Neurobiol 26:81-86

33. Kleindienst A, McGinn MJ, Harvey HB, Colello RJ, Hamm RJ, Bullock MR (2005) Enhanced hippocampal neurogenesis by intraventricular S100B infusion is associated with improved cognitive recovery after traumatic brain injury. J Neurotrauma 22:645-655

34. Sen E, Basu A, Willing LB, Uliasz TF, Myrkalo JL, Vannucci SJ, Hewett SJ, Levison SW (2011) Pre-conditioning induces the precocious differentiation of neonatal astrocytes to enhance their neuroprotective properties. ASN Neuro 3(3):e00062

35. Shao G, Lu GW (2012) Hypoxic preconditioning in an autohypoxic animal model. Neurosci Bull 28(3):316-320

36. Wick A, Wick W, Waltenberger J, Weller M, Dichqans J, Schulz JB (2002) Neuroprotection by hypoxic preconditioning requires sequential activation of vascular endothelial growth factor receptor and Akt. J Neurosci 22(15):6401-6407

37. Li YN, Pan R, Qin XJ, Yang WL, Qi Z, Liu W, Liu KJ (2014) Ischemic neurons activate astrocytes to disrupt endothelial barrier via increasing VEGF expression. J Neurochem 129(1):120-129

38. Chen CY, Sun WZ, Kang KH, Chou HC, Tsao PN, Hsieh WS, Fu WM (2015) Hypoxic preconditioning suppresses glial activation and neuroinflammation in neonatal brain insults. Mediators Inflamm 2015:632592. doi:10.1155/2015/632592 Epub 2015 Jul 27

39. Tang Y, Pacary E, Freret T, Divoux D, Petit E, Schumann-Bard $\mathrm{P}$, Bernaudin M (2006) Effect of hypoxic preconditioning on brain genomic response before and following ischemia in the adult mouse: identification of potential neuroprotective candidates for stroke. Neurobiol Dis 21(1):18-28

40. Tang E, Chen Y, Luo Y (2014) Dexamethasone for the prevention of acute mountain sickness: systematic review and metaanalysis. Int J Cardiol 173(2):133-138 